Revista Destaques Acadêmicos, Lajeado, v. 11, n. 3, 2019. ISSN 2176-3070

DOI: http://dx.doi.org/10.22410/issn.2176-3070.v11i3a2019.2314

http://www.univates.br/revistas

\title{
CONHECIMENTO E ACEITAÇÃO DOS MEDICAMENTOS GENÉRICOS POR USUÁRIOS: UMA REVISÃO INTEGRATIVA
}

\author{
Sâmia Nadine Bucco Sebben ${ }^{1}$, Luciana Carvalho Fernandes²
}

Resumo: A Política Nacional de Medicamentos (PNM) objetivou garantir a segurança, eficácia e qualidade dos medicamentos, racionalizar seu uso e garantir o acesso da população aos considerados essenciais. Nela está inclusa a promoção do uso dos medicamentos genéricos, envolvendo a produção, comercialização, prescrição e uso, para consolidá-los no Brasil. Mesmo com a implantação da PNM e com quase 20 anos no mercado brasileiro, percebe-se que medicamentos genéricos não são conhecidos, aceitos e utilizados por parte da população. O presente trabalho objetivou realizar uma revisão integrativa sobre o conhecimento dos usuários acerca dos medicamentos genéricos, analisando se os mesmos sabem diferenciar os genéricos dos de referência e similares. Os dados foram coletados através de artigos selecionados em bases de dados, perfazendo um total 12 artigos científicos, publicados entre 2005 e 2017. Foi possível concluir que houve crescente conhecimento, aceitação e uso dos genéricos ao longo do anos, devido às ações de propaganda que estimulam a propagação do conhecimento, sendo o médico uma peça chave que auxilia na escolha do tipo de medicamento.

Palavras-chave: Atenção Primária à Saúde. Conhecimentos sobre medicamentos genéricos. Medicamentos genéricos. Revisão integrativa. Uso de medicamentos.

\section{INTRODUÇÃO}

Entre as ações instauradas pelogoverno para o auxiliar o desenvolvimento da Saúde Pública do Brasil, está a implantação de Políticas de Saúde em diversas áreas de atuação. Nesse sentido, através da Portaria $n^{\circ}$ 3.916, de 30 de outubro de 1998, houve a implantação da Política Nacional de Medicamentos (PNM), com o propósito de garantir a segurança, eficácia e qualidade necessária dos medicamentos, promover seu uso racional e garantir o acesso da população aos medicamentos essenciais. A PNM tem como principais diretrizes a

1 Acadêmica do curso de Farmácia. Univates. E-mail: samia.sebben@universo.univates.br

2 Farmacêutica. Mestre em Ciências Farmacêuticas. Professora do Centro de Ciências Biológicas e da Saúde, Univates. E-mail: lufernandes@univates.br 
adoção da relação de medicamentos essenciais, regulamentação sanitária dos medicamentos, reorientação da assistência farmacêutica, promoção do uso racional de medicamentos, desenvolvimento científico e tecnológico, promoção da produção de medicamentos, garantia da segurança, eficácia e qualidade dos medicamentos, e o desenvolvimento e capacitação dos recursos humanos. Dentre as ações desenvolvidas na PNM, está inclusa a promoção do uso dos medicamentos genéricos, envolvendo a produção, comercialização, prescrição e o uso dos mesmos, realizando fiscalizações que assegurem a padronização de embalagens, para consolidação de seu uso no Brasil (BRASIL, 2001).

No Brasil, a discussão sobre os medicamentos genéricos iniciou na década de 70, porém foi só na década de 90 que houve a primeira regulamentação através do Decreto 793, de 05/04/1993 que posteriormente foi revogado pelo Decreto 3.181, de 23/09/1999, que regulamentou a Lei n ${ }^{\circ} 9.787$, de 10/02/1999. Foi através da promulgação desse decreto, que foram criadas as condições de produção e comercialização dos medicamentos genéricos no Brasil. Os genéricos só podem chegar até o consumidor depois de passarem por testes de equivalência farmacêutica e bioequivalência, comprovando sua eficácia, segurança e qualidade, o que permite que sejam intercambiáveis. Os primeiros 182 registros para produtos genéricos foram concedidos no ano 2000, quando também foram tomadas ações que implementassem sua produção, inclusive incentivando sua importação (BRASIL, 1999; ANVISA, 2018).

Mesmo com a implantação da PNM e com quase 20 anos no mercado brasileiro, percebe-se na prática que medicamentos genéricos não são conhecidos e consequentemente ainda não são aceitos e utilizados por parte da população. Com o presente trabalho, objetivou-se realizar uma revisão integrativa sobre o conhecimento dos usuários acerca dos medicamentos genéricos, analisando se os mesmos sabem diferenciar os medicamentos genéricos dos de referência e similares.

\section{MÉTODO}

Trata-se de um estudo com coleta de dados realizada a partir de fontes secundárias, por meio de levantamento bibliográfico. Para o levantamento dos artigos na literatura, foram realizadas buscas nas seguintes bases de dados: Literatura Latino-Americana e do Caribe em Ciências da Saúde (LILACS), Medical Literature Analysis and Retrieval Sistem on-line (Medline), Science Direct, Portal de Periódicos CAPES/MEC e SciELO (Scientific Electronic Library Online).

\subsection{Descritores utilizados}

Os descritores utilizados no presente trabalho - todos presentes no DeCS e MESH - foram: uso de medicamentos genéricos (Generic drug utilization, utilizado plural e singular, grafia com " $\mathrm{s}$ " e " $\mathrm{z}$ "); medicamentos (Medications); 
Atenção Primária à Saúde (Primary health care e os sinônimos: "primary care", "primary health"); conhecimento sobre medicamentos genéricos (Knowledge about generic drugs); Pesquisa qualitativa (Qualitative research, bem como "qualitative study", "qualitative studies", "qualitative analysis", "qualitative methods").

\subsection{Critérios de inclusão e exclusão}

Critérios de inclusão:

- Artigos que apresentaram no mínimo: o tipo de estudo, a abordagem, população, técnicas e instrumentos de coleta de dados;

- Artigos publicados em qualquer ano;

Critérios de exclusão:

- Artigos repetidos, sendo mantida apenas a primeira versão identificada;

- Artigos que não possuíam relação direta com o tema;

- Artigos cuja descrição metodológica apresentou informações insuficientes para o leitor entender o processo de pesquisa.

\section{RESULTADOS}

A amostra final desta revisão integrativa foi constituída por 12 artigos científicos, publicados entre os anos de 2005 e 2017, selecionados por meio dos critérios de inclusão previamente estabelecidos, conforme Figura 1. 
Figura 1 - Fluxograma da seleção dos artigos incluídos na revisão.

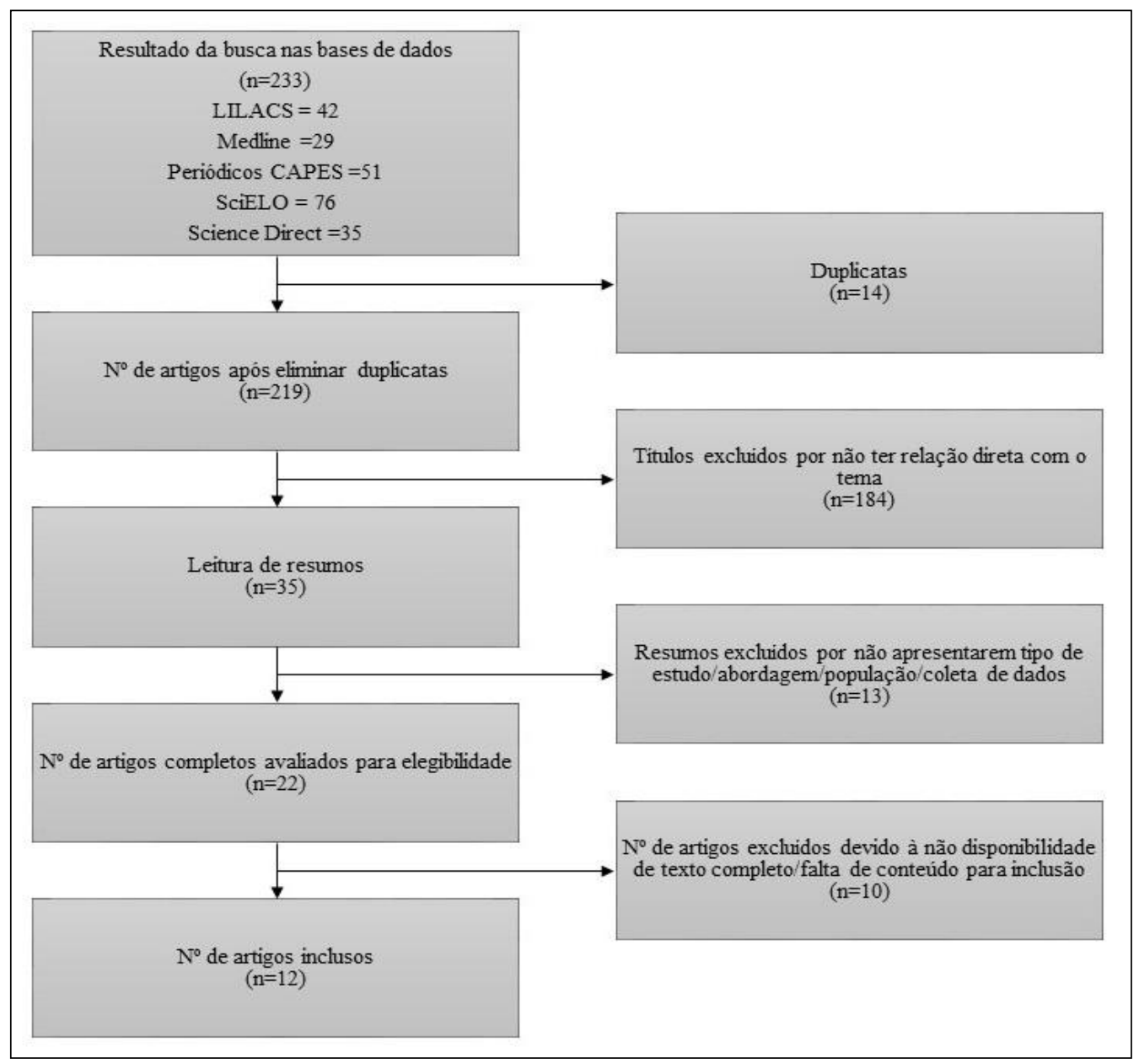

\subsection{Caracterização dos artigos avaliados}

O primeiro estudo (transversal) foi realizado por Bertoldi et al. (2005) na cidade de Pelotas/RS, com uma amostra total de 3.182 indivíduos, onde foram analisados três parâmetros diferentes: a proporção de genéricos no total de medicamentos utilizados nos 15 dias anteriores à entrevista, o conhecimento teórico e prático sobre genéricos e as estratégias para a escolha de medicamentos na compra com prescrições médicas. Para análise do conhecimento dos genéricos, foram feitos três questionamentos: "O medicamento genérico custa mais, igual ou menor que o medicamento de referência?" "A qualidade dos medicamentos genéricos é melhor, igual ou pior que a dos medicamentos de referência?" "O que contém a embalagem de medicamentos genéricos que a diferencia de outras drogas?" Também foram utilizadas imagens de 
embalagens de medicamentos de referência, genéricos e similares para analisar o reconhecimento dos entrevistados (BERTOLDI et al., 2005).

Em 2006 na cidade Rio Branco/AC, foi realizado um estudo transversal composto por 140 indivíduos de ambos os sexos, onde foi analisado o conhecimento popular sobre medicamentos genéricos por meio de um kit com oito apresentações de medicamentos de referência e genéricos, sendo considerado um ponto para cada acerto (FARIA et al., 2006).

Em 2007, Da Silva et al. analisaram o conhecimento de um grupo de pacientes com alta hospitalar de uma Santa Casa do interior de Minas Gerais sobre os medicamentos genéricos utilizados por eles. $\mathrm{O}$ estudo quantitativodescritivo entrevistou 25 indivíduos adultos (18 a 91 anos), de ambos os sexos. A pesquisa avaliou por meio de três opções o grau de conhecimento de seis quesitos: nome do medicamento genérico do qual faz uso, dose, frequência, horário de administração, efeitos esperados e efeitos colaterais (DA SILVA et al., 2007).

Ainda em 2007 foi publicado um estudo de prevalência realizado com 400 indivíduos em Recife, que analisou o grau de conhecimento e informação dos usuários acerca dos genéricos, através de um questionário. A maior parte dos entrevistados era do sexo feminino $(76,1 \%), 41,3 \%$ tinham idade superior a 50 anos, 51,4\% possuíam renda superior a um salário mínimo, mas 69,3\% não trabalhava; 63\% tinham até nove anos de estudo (ROCHA et al., 2007).

Em 2011, Vosgerau et al. analisaram a utilização de genéricos em uma área de atuação da Equipe de Saúde da Família no município de Ponta Grossa, onde foram entrevistados 374 indivíduos com idade média de 39,5 anos, com maioria do sexo feminino (53,5\%) sendo que $2,1 \%$ eram analfabetos e $43,6 \%$ não tinham ensino fundamental completo (VOSGERAU et al., 2011).

Em 2012, Blatt et al. realizaram um estudo transversal na cidade de Tubarão, com um total de 234 indivíduos, sendo a maior parte do sexo feminino $(75,6 \%)$, integrante da classe C, com idade e escolaridade média de 45,9 e 8,2 anos, respectivamente (BLATT et al. 2012).

Em 2014, Lira et al. avaliou o nível de conhecimento e percepções dos medicamentos genéricos entre leigos, por meio de um estudo transversal com 278 participantes, sendo 180 mulheres e 98 homens, com idade de 37,1 $\pm 15,8$ anos. Os participantes tiveram seu conhecimento e percepção sobre os medicamentos genéricos avaliado por meio de um questionário (LIRA et al., 2014).

Duque et al. (2014) realizou um estudo com 375 participantes, em sua maioria do sexo feminino $(65,9 \%)$, com baixo grau de escolaridade $(62,1 \%)$ (DUQUE et al. 2014).

Em 2011, Sewell et al. realizaram um estudo qualitativo em dois condados do Black Belt no Alabama com 30 indivíduos predominantemente mulheres $(n=28)$, maiores de 18 anos, que fizessem uso contínuo de pelo 
menos um medicamento para hipertensão, diabetes ou outra doença crônica. Para coletar os dados, os participantes foram divididos em quatro grupos $(\mathrm{n}=7, \mathrm{n}=9, \mathrm{n}=6, \mathrm{n}=8)$, e cada grupo realizou uma discussão sobre seis temas (diferenças percebidas na eficácia; diferenças percebidas nos efeitos colaterais; percepções de que os genéricos não são medicamentos reais; disposição para usar genéricos para doenças menores, mas não graves; confiança no sistema médico; percepção de "ter que se contentar" com medicamentos genéricos se você é pobre) que durou de 30 a 60 minutos. $43 \%$ dos participantes tinha ensino superior completo, $13,33 \%$ não concluiu nem o ensino médio; mais de $40 \%$ dos participantes tomava 6 ou mais medicamentos por dia (SEWELL et al., 2011).

Em 2012, Guttier et al..replicou o estudo de Bertoldi para verificar a percepção, conhecimento, e uso de medicamentos genéricos em usuários da cidade de Pelotas e entrevistou 2.925 pessoas (GUTTIER et al., 2016).

Em 2016, Monteiro et al. publicou um estudo transversal que analisou o uso de genéricos pela população com diabetes mellitus (DM) e hipertensão arterial sistêmica (HAS) na cidade de São Paulo, em 2003. A pesquisa englobou 603 pessoas, sendo que 448 dos entrevistados relataram ser hipertensos e 155 ser diabéticos, o total dos entrevistados referiu ter utilizado medicamento para controle de HAS, DM ou outra enfermidade nos três dias que antecederam a entrevista. Foram analisadas as variáveis utilização de medicamentos genéricos e percepção sobre tais medicamentos. A utilização dos medicamentos genéricos foi avaliada através do questionamento "Está usando medicamento genérico?" E a percepção foi analisada por meio das perguntas: “O (a) $\operatorname{sr}(a)$ sabe se é possível substituir algum dos medicamentos que usou nos últimos três dias por um genérico?", "Na sua opinião, existem vantagens em utilizar medicamentos genéricos? Quais?" e "Na sua opinião, existem desvantagens em utilizar medicamentos genéricos? Quais?" (MONTEIRO et al., 2016)

O Quadro 1 apresenta a descrição dos artigos analisados na presente revisão.

Quadro 1: descrição dos artigos incluídos na presente revisão

\begin{tabular}{|l|l|l|l|}
\hline Título & Ano & Delineamento & Objetivo \\
\hline $\begin{array}{l}\text { Generic drugs in Brazil: } \\
\text { known by many, used } \\
\text { by few }\end{array}$ & 2005 & $\begin{array}{l}\text { Transversal } \\
\text { de base } \\
\text { populacional }\end{array}$ & $\begin{array}{l}\text { Avaliar o conhecimento e uso } \\
\text { de genéricos em uma amostra } \\
\text { populacional de adultos de uma cidade } \\
\text { do sul do Brasil }\end{array}$ \\
\hline $\begin{array}{l}\text { Conhecimento popular } \\
\text { sobre medicamento } \\
\text { genérico em um Distrito } \\
\text { Docente-Assistencial } \\
\text { do Município de Rio } \\
\text { Branco, Estado do Acre, } \\
\text { Brasil }\end{array}$ & 2006 & Transversal & $\begin{array}{l}\text { Avaliar o conhecimento popular sobre } \\
\text { medicamentos genéricos utilizando } \\
\text { amostra probabilística da população } \\
\text { do Distrito Docente-Assistencial do } \\
\text { Tucumã, no Município de Rio Branco, } \\
\text { Estado do Acre, Brasil }\end{array}$ \\
\hline
\end{tabular}




\begin{tabular}{|c|c|c|c|}
\hline Título & Ano & Delineamento & Objetivo \\
\hline $\begin{array}{l}\text { Conhecimento de um } \\
\text { grupo de pacientes sobre } \\
\text { medicamentos genéricos } \\
\text { por eles utilizados }\end{array}$ & 2007 & $\begin{array}{l}\text { Quantitativo } \\
\text { descritivo }\end{array}$ & $\begin{array}{l}\text { Analisar o conhecimento de um grupo } \\
\text { de pacientes de uma Santa Casa do } \\
\text { interior do Estado de Minas Gerais } \\
\text { acerca dos medicamentos genéricos por } \\
\text { eles utilizados }\end{array}$ \\
\hline $\begin{array}{l}\text { Levantamento de dados } \\
\text { sobre o conhecimento e } \\
\text { informação acerca dos } \\
\text { medicamentos genéricos } \\
\text { em uma população de } \\
\text { pacientes do serviço de } \\
\text { saúde ambulatorial do } \\
\text { Recife, Pernambuco, } \\
\text { Brasil }\end{array}$ & 2007 & Transversal & $\begin{array}{l}\text { Verificar o grau de conhecimento e } \\
\text { informação sobre os medicamentos } \\
\text { genéricos, em amostra representativa } \\
\text { de usuários do serviço público } \\
\text { de saúde ambulatorial do Recife, } \\
\text { Pernambuco, Brasil e sua associação } \\
\text { com o perfil socioeconômico desses } \\
\text { usuários }\end{array}$ \\
\hline $\begin{array}{l}\text { Perceptions of and } \\
\text { barriers to use of } \\
\text { generic medications in } \\
\text { a rural african american } \\
\text { population, Alabama, } \\
2011\end{array}$ & 2011 & Qualitativo & $\begin{array}{l}\text { Analisar qualitativamente a visão } \\
\text { das crenças sobre o uso genérico de } \\
\text { medicamentos entre afro-americanos } \\
\text { no sul rural }\end{array}$ \\
\hline $\begin{array}{l}\text { Utilização de genéricos } \\
\text { em área de atuação da } \\
\text { equipe de Saúde da } \\
\text { Família em município do } \\
\text { sul do Brasil }\end{array}$ & 2011 & Transversal & $\begin{array}{l}\text { Estimar a prevalência do uso de } \\
\text { genéricos na população total e entre os } \\
\text { usuários de medicamentos; verificar } \\
\text { as classes medicamentosas mais } \\
\text { consumidas entre os genéricos; avaliar } \\
\text { o nível de conhecimento dos genéricos } \\
\text { pela população; e identificar os fatores } \\
\text { associados ao uso de genéricos entre } \\
\text { adultos de } 20 \text { a } 59 \text { anos. }\end{array}$ \\
\hline $\begin{array}{l}\text { Conhecimento popular } \\
\text { e utilização dos } \\
\text { medicamentos genéricos } \\
\text { na população do } \\
\text { município de Tubarão, } \\
\text { SC }\end{array}$ & 2012 & Transversal & $\begin{array}{l}\text { Pesquisar o nível de conhecimento e } \\
\text { o grau de utilização de genéricos em } \\
\text { residentes do município de Tubarão, } \\
\text { SC }\end{array}$ \\
\hline $\begin{array}{l}\text { Conhecimento, } \\
\text { percepções e utilização } \\
\text { de medicamentos } \\
\text { genéricos: um estudo } \\
\text { transversal }\end{array}$ & 2014 & Transversal & $\begin{array}{l}\text { Avaliar nível de conhecimento, } \\
\text { percepções e perfil de utilização dos } \\
\text { medicamentos genéricos entre leigos }\end{array}$ \\
\hline $\begin{array}{l}\text { Adesão dos utentes aos } \\
\text { medicamentos genéricos }\end{array}$ & 2014 & Transversal & $\begin{array}{l}\text { Caracterizar a adesão dos utentes aos } \\
\text { medicamentos genéricos, bem como } \\
\text { identificar fatores que influenciam tal } \\
\text { adesão }\end{array}$ \\
\hline $\begin{array}{l}\text { Percepção, conhecimento } \\
\text { e uso de medicamentos } \\
\text { genéricos no Sul do } \\
\text { Brasil: o que mudou } \\
\text { entre } 2002 \text { e } 2012 ?\end{array}$ & 2016 & $\begin{array}{l}\text { Transversal } \\
\text { de base } \\
\text { populacional }\end{array}$ & $\begin{array}{l}\text { Descrever as mudanças na utilização } \\
\text { de medicamentos genéricos bem } \\
\text { como na percepção e conhecimento da } \\
\text { população sobre os mesmos, após } 12 \\
\text { anos da entrada desses medicamentos } \\
\text { no mercado farmacêutico brasileiro. }\end{array}$ \\
\hline
\end{tabular}




\begin{tabular}{|l|l|l|l|}
\hline Título & Ano & Delineamento & Objetivo \\
\hline $\begin{array}{l}\text { Utilização de } \\
\text { medicamentos genéricos } \\
\text { no município de São } \\
\text { Paulo em 2003: estudo } \\
\text { de base populacional }\end{array}$ & 2016 & Transversal & $\begin{array}{l}\text { Analisar a utilização e a percepção } \\
\text { sobre medicamentos genéricos pela } \\
\text { população com diabetes e hipertensão } \\
\text { na cidade de São Paulo, considerando- } \\
\text { se a Política de Medicamentos } \\
\text { Genéricos no Brasil }\end{array}$ \\
\hline $\begin{array}{l}\text { Factors influencing } \\
\text { the preference for } \\
\text { purchasing generic } \\
\text { drugs in a Southern } \\
\text { Brazilian city }\end{array}$ & 2017 & $\begin{array}{l}\text { Transversal } \\
\text { de base } \\
\text { populacional }\end{array}$ & $\begin{array}{l}\text { Identificar fatores associados } \\
\text { à preferência pela compra de } \\
\text { medicamentos genéricos em um } \\
\text { município de médio porte do sul do } \\
\text { Brasil }\end{array}$ \\
\hline
\end{tabular}

\subsection{Conhece e/ou sabe identificar os medicamentos genéricos}

O estudo de Bertoldi et al. mostrou que os participantes possuíam algum conhecimento sobre os genéricos pois $86 \%$ disseram custar menos que o de referência, $70 \%$ acredita que a qualidade de ambos seja equivalente, 56,6\% poderia identificar determinadas características da embalagem dos genéricos que os diferenciam dos outros. Sobre o reconhecimento das embalagens, $48 \%$ indicou erroneamente que o medicamento similar era genérico, enquanto apenas $12 \%$ classificou inversamente. A identificação incorreta de similares como genéricos aumentou significativamente com a idade e diminuiu com a elevação do grau de escolaridade e status econômico (BERTOLDI et al., 2005).

No estudo de Faria et al., 22,1\% dos participantes não soube definir com precisão o que são os medicamentos genéricos, além de não saber diferenciálo pelas embalagens apresentadas no kit. Cerca de $85 \%$ dos entrevistados souberam reconhecer através de características citadas qual era o genérico entre as apresentações (FARIA et al., 2006).

Analisando a porcentagem de conhecimento vista por Da Silva et al., os participantes relataram conhecer $76 \%(\mathrm{n}=19)$ o nome da medicação, $60 \%$ $(n=15)$ a dose, $80 \%(n=20)$ a frequência, $84 \%(n=21)$ o horário, $56 \%(n=14)$ o efeito esperado e $4 \%(n=1)$ os efeitos colaterais. Percebeu-se que a maioria dos participantes conhece seus medicamentos, porém, tal conhecimento é inversamente proporcional quando se tratam dos efeitos colaterais, visto que $84 \%(n=21)$ relatou desconhecer tais informações. Já sobre o conhecimento de genéricos em geral, os participantes disseram: $56 \%$ não conhecer, $8 \%$ ser o mesmo que o de referência, ter o mesmo efeito e menor custo, $28 \%$ ser o medicamento mais barato e $8 \%$ ser mais barato e mais fraco; $100 \%$ dos participantes relatou não saber a diferença entre os medicamentos de referência, genérico e similar. Tal fato deve-se às poucas informações disponíveis sobre o assunto em 2007 e ao baixo nível de escolaridade dos participantes (DA SILVA et al., 2007).

A pesquisa de Rocha et al. mostrou que 95,7\% dos entrevistados já ouviu falar sobre os genéricos, $68,1 \%$ soube identificá-lo e diferenciá-lo do 
medicamento de referência e $65,3 \%$ sabia defini-lo. Entre os indivíduos que souberam definir, $40 \%$ disseram ser aquele que custa menos que o de referência. $47 \%$ elencou a letra $G$ na embalagem como forma de identificação, enquanto $25 \%$ disse identificar através da letra $G$ presente na tarja amarela, concluindo que $91,5 \%$ dos participantes eram capazes de reconhecer o genérico. Quando comparadas as formas de identificação e definição dos genéricos segundo custo e composição, foi possível identificar que $69,8 \%$ dos participantes disseram que o genérico têm custo menor que o de referência, ao passo que, somente $27,8 \%$ disse reconhecê-lo através do preço, $46,7 \%$ dos entrevistados disseram que o genérico possuía a mesma composição do medicamento de referência, enquanto apenas 19,4\% reconheceu o mesmo pela substância ativa (ROCHA et al., 2007).

Vosgerau et al. verificou que $96,5 \%$ dos participantes relatou ter conhecimento sobre os medicamentos genéricos. Em se tratando da qualidade destes, a maioria $(64,3 \%)$ relatou possuir a mesma qualidade, $22,7 \%$ disse não saber, $11,1 \%$ respondeu ter menor qualidade e $1,9 \%$ disse ter melhor qualidade. Em relação aos preços, a maioria $(88,9 \%)$ disse que os genéricos são produtos mais baratos (VOSGERAU et al., 2011).

Blatt et al. verificou como os entrevistados identificavam os genéricos, $37,6 \%$ identifica pela letra "G", 28,2\% pela "tarja amarela" e 23,9\% pela inscrição "Medicamento Genérico". Após a apresentação de seis ilustrações (referência, genérico e similar de paracetamol e atenolol), foi solicitado que os entrevistados identificassem as imagens. $91 \%$ identificou corretamente os dois exemplos de genéricos; enquanto apenas $25,6 \%$ identificou de maneira correta as seis ilustrações (BLATT et al., 2012).

Dentre os entrevistados por Lira et al., 99,6\% já ouviram falar dos genéricos. Cerca de 48,6\% definiram corretamente os genéricos e 30,9\% não soube responder. Do total de 278 entrevistados, 219 (78,8\%) relataram ter recebido informações sobre os genéricos através dos meios da televisão $(49,3 \%)$, médicos $(18 \%)$, em farmácias $(39,5 \%)$, internet e/ou jornais $(7,2 \%)$, conhecidos ou vizinhos $(3,6 \%)$, rádio $(2,9 \%)$, propagandas de rua $(1,8 \%)$ e outros profissionais da saúde e universidades $(0,7 \%)$; do mesmo total, $81 \%$ relataram que utilizam ou já utilizaram genéricos (LIRA et al., 2014).

Duque et al. verificou que $98,7 \%$ disseram já ter ouvido falar sobre os genéricos; destes, $78,9 \%$ já havia adquirido tais medicamentos. Entre os que já compraram, todos acreditam que tais produtos sejam mais baratos do que os de referência e 62,9\% considera ter a mesma qualidade (DUQUE et al., 2014).

Quanto ao reconhecimento das embalagens dos medicamentos, Guttier et al. verificou que apenas $33 \%$ dos participantes identificou erroneamente o genérico como sendo similar, o que levou a uma redução de $15 \%$ quando relacionado aos dados do primeiro estudo (48\%). Em relação ao conhecimento sobre os genéricos, os dados não tiveram aumento significativo nestes 10 anos. Em 2002, 86\% dos entrevistados disseram que os medicamentos genéricos 
custam menos que os de referência, enquanto em 2012 esse percentual foi de $87 \%$. Os que acreditavam que a qualidade de medicamentos de referência e do genérico era equivalente somaram 70\% em 2002 e 69,1\% em 2012 (GUTTIER et al., 2016).

Segundo os dados apresentados por Monteiro et al., houve utilização de genéricos e grande falta de conhecimento acerca da possibilidade de substituição do medicamento de referência pelo genérico. Não foi identificada diferença significativa quanto a utilização de genéricos segundo idade, sexo e grau de escolaridade (MONTEIRO et al., 2016).

No estudo de Guttier et al., $88,1 \%$ dos entrevistados tinham conhecimento de que os genéricos eram mais baratos que os de referência, $69,8 \%$ relatou saber que possuem qualidade equivalente, e $76,6 \%$ foram capazes de identificar corretamente as características relativas à embalagem que diferenciam os genéricos. Para a análise do reconhecimento da embalagem, foi apresentada uma imagem de um medicamento de referência e após um similar, com posterior questionamento se este era o genérico, $72 \%$ dos entrevistados disseram não ser o medicamento genérico e $28 \%$ disseram ser (GUTTIER et al., 2017).

\subsection{Utiliza/prefere/confia em medicamentos genéricos}

A prevalência da utilização de genéricos encontrada por Bertoldi et al. foi de aproximadamente $4 \%$, de acordo com a amostra de 3.182 indivíduos. A utilização foi vista de acordo com diversas variáveis, tendo como resultado que os medicamentos de referência eram usados mais pelos jovens, de classe econômica elevada e mais instruídos. Produtos similares eram o oposto, preferidos por adultos de meia-idade, de classe econômica mais baixa e menos instruídos. Genéricos raramente foram utilizados e não houve diferença em relação às variáveis do estudo. 30,4\% dos indivíduos havia adquirido medicamentos com receita médica nos últimos 15 dias; $7,7 \%$ adquiriu exatamente o que estava na prescrição; $10,6 \%$ adquiriu a forma genérica do medicamento prescrito e $4,1 \%$ comprou um produto mais barato, independente de ser genérico, similar ou manipulado (BERTOLDI et al. 2005).

Já Sewell et al., analisando a eficácia de medicamentos genéricos e de referência, em diferentes grupos verificou que os genéricos foram elencados como menos eficazes ou potentes do que os de referência, sendo que alguns relataram que se pudessem pagar, comprariam medicamentos de referência, pois estes são mais poderosos do que os genéricos. Quando discutiram sobre os efeitos colaterais, os participantes alegaram que os genéricos possuem mais efeitos colaterais, pois é necessário que se use uma dose maior para ter o efeito desejado. Tal opinião esteve presente nas discussões assim como a crença de que os genéricos não são medicamentos reais. Os participantes mostraram-se dispostos a utilizar medicamentos genéricos somente para doenças que não 
fossem tão graves, como alergias e resfriados, não empregando por exemplo, em casos de hipertensão. As maiores barreiras encontradas no estudo são as crenças sobre medicamentos, de que estes têm menor eficácia e segurança, o que contribui para a subutilização dos genéricos (SEWELL et al., 2011).

No estudo proposto por Vosgerau et al. (2011), analisando a preferência por medicamentos, foi possível constatar que $60,7 \%$ prefere genéricos, $13,9 \%$ prefere medicamento de referência, 1,9\% similares e $23,5 \%$ disse não se importar. Nos sete dias que antecederam a entrevista, a prevalência de consumo de medicamentos foi de $67,1 \%(n=251)$, entre estes, a prevalência do uso de genéricos foi de $14,7 \%$. Ao considerar os 374 entrevistados, a proporção de entrevistados que utilizaram pelo menos um produto genérico na última semana foi de 9,9\% (VOSGERAU et al., 2011).

Quando questionados sobre a confiança nos medicamentos genéricos, $76,9 \%$ dos entrevistados por Blatt et al. acreditam ter o mesmo efeito do medicamento de referência e quase $90 \%$ dos que já fizeram uso de genéricos mostraram-se satisfeitos com o resultado. A maior parte dos participantes já comparou a diferença de preço entre os medicamentos de referência e genérico, sendo o segundo mais barato em $97 \%$ dos casos. $74,4 \%$ dos participantes relatou que entre os dois medicamentos, escolheria o genérico (BLATT et al., 2012).

Os entrevistadores questionaram se os participantes possuíam medicamentos genéricos em casa, aos que afirmaram, foi solicitada sua caixa de medicamentos para conferência. Em $75,6 \%$ dos casos, houve coerência entre o produto encontrado e a embalagem que acreditavam ser um genérico. Aproximadamente $85 \%$ dos entrevistados já fizeram uso de genéricos, dentre estes, $77,8 \%$ adquirem com frequência. Em cerca de $69,2 \%$ dos casos, o usuário foi o responsável pela solicitação do medicamento, 37,4\% foi indicação do farmacêutico, 19,8\% prescrição médica e 1,1\% obtenção do SUS (BLATT et al., 2012).

A maioria dos entrevistados por Lira et al. relatou confiar na eficácia dos genéricos $(79,1 \%)$, acreditar que possuem o mesmo efeito $(74,8 \%)$ e segurança $(75,2 \%)$ dos de referência, porém, $14,4 \%$ dos entrevistados relataram achar que os genéricos têm qualidade inferior a do de referência. $88,8 \%$ relataram que o genérico tem custo menor que o medicamento de referência e $80,2 \%$ afirmaram adquirir o genérico por conta do preço. $56,5 \%$ afirmaram que os genéricos possuem a mesma substância que o de referência e 50,3\% consideraram boa a divulgação dos genéricos no Brasil; 43,2\% afirmaram encontrar genéricos nas farmácias. Quando questionados sobre a troca do medicamento de referência pelo genérico quando ofertado pelo farmacêutico, $65 \%$ responderam que sim, caso fosse mais barato; apenas $26,6 \%$ aceitariam a troca, caso fosse o mesmo preço e $34,2 \%$ não optariam pelo genérico em detrimento do de referência (LIRA et al., 2014).

Na pesquisa realizada por Guttier et al. verificou-se que a utilização de medicamentos genéricos aumentou consideravelmente entre 2002 e 2012, 
somando $65,9 \%$ e $76,6 \%$, respectivamente. No primeiro estudo foi identificada a prevalência de utilização de genéricos de 3,6\%, sendo que em 2012 tal dado aumentou para $26,1 \%$. No ano de 2002 , não houve diferença na percepção da qualidade dos genéricos entre indivíduos que utilizaram e os que não o utilizaram. Já em 2012, houve diferença significativa na percepção da qualidade dos genéricos entre os mesmos grupos, sendo maior a percepção de que o genérico tem qualidade equivalente entre os que utilizaram tal produto, do que entre aqueles que não o fizeram. O emprego de genéricos em Pelotas foi sete vezes maior em 2012 quando comparado a 2002. Houve importante crescimento na habilidade de diferenciação dos medicamentos genérico e similar, assim como no reconhecimento das características da embalagem. Também observou-se aumento estatisticamente significativo do número de indivíduos que substituíram o medicamento prescrito pelo genérico no ato da compra. Em se tratando dos participantes que possuíam conhecimento sobre os genéricos, $77,7 \%$ preferiram adquiri-los, enquanto tal proporção foi de apenas $18,4 \%$ entre os participantes sem bom conhecimento. Os participantes que responderam corretamente às três perguntas estavam mais predispostos à optar pelos genéricos. Aqueles que tinham conhecimento de que o genérico era mais barato que o de referência tinham maior preferência pela aquisição de genéricos (51\%) do que os que desconheciam tal informação. Os que conheciam que a qualidade de ambos os medicamentos é equivalente, tinham $115 \%$ mais de chances de optar pelos genéricos do que aqueles que não sabiam. Aqueles que também souberam identificar corretamente os aspectos que diferenciam os genéricos, também tinham preferência por estes (GUTTIER et al., 2016).

Dentre os entrevistados de Monteiro et al. que referiram HAS ou DM, fizeram uso de medicamentos nos três dias anteriores à entrevista e eram usuários de genéricos, 33,3\% (n=135) eram hipertensos e $26,3 \%(n=49)$ eram diabéticos. Dos que referiram HAS, 22,7\% não conheciam a possibilidade de substituição do medicamento. Para os que referiram DM, 22,2\% desconhecia tal possibilidade (MONTEIRO et al., 2016).

\subsection{Gênero e faixa etária}

Os usuários que mais reconheceram os genéricos no estudo de Faria et al. foram os homens (36,4\%), enquanto a proporção entre mulheres é de apenas $12,9 \%$. Em relação à faixa etária dos 31 participantes que reconheceram, foi evidenciada uma média de idade de $36,9 \pm 10,1$ anos, sendo semelhante à idade média (38,2 $\pm 10,3$ anos) das 109 pessoas que não possuíam tal conhecimento (FARIA et al., 2006).

Rocha et al. evidenciaram que gênero, faixa etária e classe econômica/ renda não apresentaram associações significantes, visto que em ambas classes o conhecimento foi percebido de maneira similar (ROCHA et al., 2007). 
Duque et al. (2014) concluiu que os participantes mais novos têm prevalência elevada na compra de genéricos por solicitação ao farmacêutico $(47,3 \%)$, enquanto $93,2 \%$ dos idosos compram tais produtos com prescrição médica (DUQUE et al., 2014).

No estudo de Guttier et al. foi possível identificar que entre os entrevistados que preferiram os genéricos $(63,2 \% \mathrm{n}=1.804)$, usuários do sexo masculino tiveram maior preferência $(61,04 \%)$, quando comparados às participantes do sexo feminino $(38,96 \%)$. Com relação à idade, constatou-se que o aumento da idade é inversamente proporcional à preferência (20-39 anos = $42,13 \%$; $40-59$ anos $=37,42 \%$; mais de 60 anos $=20,45 \%$ ) (GUTTIER et al., 2017).

\subsection{Classe econômica e grau de escolaridade}

Os participantes do estudo de Faria et al. foram separados em duas categorias, segundo o nível de escolaridade: básico, incluindo aqueles com $2^{\circ}$ grau incompleto; e médio ou superior. O grupo que conhecia os medicamentos foi composto por: $25,8 \%$ de nível básico e $74,2 \%$ de nível médio ou superior; enquanto o grupo "não conhecedor", $73,4 \%$ e $26,6 \%$, respectivamente (FARIA et al., 2006).

Guttier et al. observaram que a preferência por medicamentos genéricos cresceu conforme o aumento do grau de escolaridade dos participantes: $57,4 \%$ para até quatro anos de estudo, 66,5\% entre cinco e oito anos de estudo e 63,3\% para nove ou mais anos de estudo (GUTTIER et al., 2017).

\subsection{Vantagens e desvantagens do uso de medicamentos genéricos}

Monteiro et al. verificou que $91,2 \%$ dos entrevistados que relataram HAS $(n=448)$ e $90,9 \%$ dos que relataram DM ( $n=155)$, disseram encontrar vantagens no uso dos genéricos. A maior parte dos entrevistados apontou como vantagens o baixo custo ( $\cong 71,05 \%$ em ambos os grupos), citando também o aumento de opções, facilidade para encontrar o medicamento, e genéricos "serem bons". Mesmo com a maior parte dos participantes tendo elencado vantagens no uso dos genéricos, em 2003 foi identificada baixa utilização de tais medicamentos pela população hipertensa e diabética em estudo. Já as desvantagens no uso dos genéricos, $65,9 \%(n=265)$ dos que relataram HAS e $59,4 \%(n=87)$ dos que relataram DM, consideraram como desvantagem para o uso de genéricos "não ser bom como o de marca" (10,4\% portadores de HAS e $7,2 \%$ portadores de DM) e "ser difícil de encontrar" (1,8\% para portadores de HAS e 3,4\% para portadores de DM) (MONTEIRO et al., 2016). 


\section{DISCUSSÃO}

Sete estudos versaram sobre conhecimento acerca dos medicamentos genéricos enquanto cinco avaliaram a utilização desses medicamentos por diversas populações.

Os estudos foram heterogêneos em relação ao público amostrado. Foram realizados estudos com público aleatório e com grupos específicos: pacientes em alta hospitalar, portadores de diabetes mellitus e hipertensão arterial sistêmica, moradores de locais específicos, populações já estudadas anteriormente.

Foi possível constatar diferença nas variáveis escolhidas pelos estudos qualitativo-descritivo e quantitativo, em relação àquelas empregadas em estudos transversais. Sendo que a maioria dos estudos de prevalência elegeram basicamente variáveis sociodemográficas (gênero, faixa etária, grau de escolaridade e nível socioeconômico), enquanto os outros estudos abrangeram mais aspectos, como por exemplo estado civil, conhecimento sobre medicamentos que faz uso, confiança e conhecimento sobre genéricos e diferenças destes com similares e de referência; atendimentos em serviços de saúde.

Em relação ao delineamento, a maioria dos estudos foi do tipo transversal, que são aqueles que medem a prevalência de determinado fenômeno, conforme definido por Bonita et al. (2010). Também estiveram presentes, em menor número, estudos do tipo quantitativo descritivo, que são investigações de pesquisa empírica, com a finalidade de delinear ou analisar as características de fatos ou fenômenos, conforme definição de Marconi e Lakatos (2003); bem como estudos do tipo qualitativo, definidos por Gerhardt e Silveira (2009) como sendo que são aqueles que não se preocupam com a representatividade numérica, mas, sim, com o aprofundamento da compreensão de um determinado grupo. Tais pesquisas visam explicar o porquê das coisas, mostrando o que convém ser feito, sem quantificar os valores (MARCONI e LAKATOS, 2003; GERHARDT e SILVEIRA, 2009; BONITA et al., 2010).

Nos estudos mais antigos, como o de Bertoldi et al. (2005), analisouse que pode ter havido uma confusão entre genéricos e similares na época, pois muitos associavam o primeiro ao baixo preço, o que poderia induzi-los a adquirir algum produto similar, visto que muitas vezes estes são os mais baratos. Tal informação se confirma através dos dados apresentados em relação aos medicamentos utilizados para diversas situações, quando a porção de similares foi maior do que a de genéricos.

A PNM trouxe como aspectos positivos a garantia da eficácia, segurança e qualidade dos medicamentos, tornando os genéricos equivalentes aos medicamentos de referência. Posteriormente, com a publicação da RDC $\mathrm{n}^{\circ} 58$ de 10 de outubro de 2014, foram definidos os requisitos necessários para que o medicamento similar pudesse ser intercambiável com o de referência. $O$ fato de $70 \%$ dos entrevistados por Bertoldi et al. (2005) terem considerado a qualidade 
dos produtos genéricos equivalente aos medicamentos de referência, mostra que a PNM já estava bem implementada para tal quesito, no ano do estudo (BRASIL, 2014).

Em contraponto ao estudo anterior, Faria et al. (2006) observaram que apenas $22,1 \%$ dos entrevistados possuía algum conhecimento sobre os genéricos, destes, a maioria era do sexo masculino, com idade superior a 38 anos. Tal fato pode ser explicado pelo baixo nível socioeconômico ou pelo baixo grau de escolaridade da população em questão. Este estudo teve como limitações concentrar-se apenas em avaliar a opinião dos usuários sobre as campanhas de genéricos, sem identificar também o grau de confundimento entre genéricos e similares, sendo que muitos dos participantes pensavam haver dois tipos de genéricos, enquanto outros relataram estar usando estes, enquanto estavam fazendo uso de um similar, o que nos mostra que tais diferenças ainda não eram entendidas pela maior parte da população.

$\mathrm{Na}$ alta hospitalar foi possível perceber baixo conhecimento sobre tal assunto, principalmente no que se refere ao nome do medicamento genérico, efeitos esperados e efeitos colaterais. O que é preocupante, visto que os pacientes necessitam saber determinados aspectos e informações sobre sua terapia, já que muitos se orientam pela caixa do medicamento ou forma do comprimido, por exemplo, o que varia de laboratório para laboratório, para que se possa assegurar a efetividade do tratamento e impedir o desencadeamento de possíveis erros (DA SILVA et al., 2007).

O estudo de Rocha et al. (2007) concluiu que mesmo com $63 \%$ dos entrevistados tendo cursado apenas até o ensino fundamental completo ou não, a maioria dos participantes tinha bom grau de informação sobre genéricos, seja por ter ouvido falar sobre $(95,7 \%)$, conhecer ou saber o que são, mostrando também que o nível de escolaridade e idade inferior à 50 anos, estão associados positivamente à conhecer e entender o que são os genéricos.

Sewell et al. (2011) concluíram que as crenças relacionadas à segurança e eficácia dos genéricos são as maiores barreiras que dificultam o emprego destes.

Os resultados apresentados por Vosgerau et al. (2011) mostram alta taxa de conhecimento dos genéricos $(95,6 \%)$, o que indicou aumento da difusão do produto na população, quando comparado aos estudos mais antigos. Porém, notou-se baixa prevalência de uso dos mesmos (9,9\%), tal informação apontou na época, a necessidade de realizar avanços no fortalecimento da utilização de genéricos entre a população brasileira, através da priorização destes produtos na rede pública, já que esta era a principal forma de acesso à medicamentos dos participantes do estudo.

Blatt et al. (2012) perceberam que a população estudada tinha bom nível de conhecimento sobre genéricos, a ponto de identificá-los corretamente (91\%) e aceitar bem seu uso. A maioria que já havia feito uso de genéricos $(85 \%)$, aprovou-o e disse ter o mesmo efeito que o de referência, por um custo 
menor. Dentre estes que já utilizaram, 37,4\% relatou que o uso do genérico ou a substituição por ele, foi sugerida pelo farmacêutico no ato da compra. Isso mostra que a confiança e aceitação do usuário em relação ao medicamento genérico, pode ser muito mais estimulada e fortalecida por meio da experiência de uso e orientações de quem entende do assunto.

Lira et al. (2014) investigaram as percepções, conhecimento e utilização de tais medicamentos após 15 anos da promulgação da Lei 9.787/99. Observouse que, 99,6\% dos entrevistados já tinha ouvido falar deles, mostrando que o cenário brasileiro melhorou ao longo dos anos, e é melhor do que em outros países. Neste estudo, $48,6 \%$ dos entrevistados soube definir os genéricos corretamente e a maioria $(79,1 \%)$ acredita em sua eficácia. Essas informações possivelmente traduzem elevada propensão à utilização de tais produtos pela população estudada. Quando se trata da troca do medicamento de referência pelo genérico, $65,8 \%$ dos participantes aceitaria se o genérico fosse mais barato. Cerca de $73,4 \%$ não trocaria se o preço fosse o mesmo e $97,1 \%$ não aceitaria se o genérico fosse mais caro. Isso mostra que o preço do produto genérico é um fator decisivo para seu uso ou não.

Mesmo que 98,7\% dos participantes do estudo de Duque et al. (2014) tenham dito conhecer os medicamentos genéricos, é alta a porcentagem $(21,1 \%)$ de indivíduos que nunca comprou um produto deste, por conta do médico nunca ter prescrito. Além disso, $70 \%$ dos participantes relataram que não solicitam ao prescritor o produto genérico, pois consideram que esta escolha é apenas do médico. Em cerca de $40 \%$ das respostas, não foi possível definir corretamente os genéricos, sendo os participantes menos instruídos considerados menos informados em relação ao assunto.

Observou-se um crescimento do conhecimento e emprego dos produtos genéricos ao longo desses dez anos, o que serve de indicativo de que a política de tais medicamentos evoluiu conforme planejado, ampliando o acesso aos medicamentos. Entre os que mais utilizavam genéricos, a maioria eram mulheres, de maior idade e com elevado grau de escolaridade. Esses dados podem ser explicados pelo fato de que as mulheres buscam mais os serviços de saúde, tem mais preocupação com o autocuidado e sua saúde, e normalmente utilizam mais medicamentos do que os homens. Também houve avanço na habilidade de diferenciar o medicamento genérico dos demais e reconhecer características de sua embalagem. Os usuários passaram a aceitar mais a substituição do medicamento prescrito pelo genérico, o que impacta numa maior utilização desses medicamentos. Tais avanços podem ser justificados pela maior disponibilidade e visibilidade de tais produtos nos estabelecimentos de saúde, bem como por seus preços mais acessíveis (GUTTIER et al., 2016).

Em contraponto, o estudo de Monteiro et al. (2016) mostrou baixa utilização de genéricos, e que grande parte da população estudada desconhecia a possibilidade de substituição do medicamento de referência pelo genérico. Também reforçou a importância do genérico para a promoção do acesso 
universal aos medicamentos, visto que facilita o acesso por meio de preços mais baixos. Este estudo foi o único que analisou detalhadamente vantagens e desvantagens do uso dos medicamentos genéricos, relatadas pelos usuários participantes, sendo que, a maioria relatou ter vantagens no emprego de tais produtos.

\section{CONCLUSÃO}

Com o presente estudo pode-se concluir que houve aumento significativo do conhecimento, aceitação e uso dos genéricos ao longo dos anos. Tal conquista se deve às ações de propaganda para estimular a propagação do conhecimento sobre tais produtos, seja nos estabelecimentos de saúde, através dos prescritores, ou até mesmo por meio de programas do governo como o Aqui Tem Farmácia Popular, que disponibiliza medicamentos, em sua maioria $(85 \%)$ genéricos. A parcela que ainda não conhece ou utiliza os genéricos, provavelmente desconhece os testes aos quais eles são submetidos para assegurar sua eficácia, segurança e qualidade, e receberem aprovação da ANVISA. Isso pode levar à equivocadas opiniões que subestimam a qualidade do produto e ocasionam sua baixa utilização, visto que, notou-se que quanto maior o conhecimento sobre os genéricos, maior foi a preferência sobre seu uso. O médico é um elo importante pois boa parte da população imputa a decisão de escolha do tipo de medicamento a esse profissional.

\section{REFERÊNCIAS}

AGÊNCIA NACIONAL DE VIGILÂNCIA SANITÁRIA. Medicamentos genéricos. Disponível em:<http:/ /portal.anvisa.gov.br/genericos>. Acesso em: 02 nov. 2018.

BERTOLDI, Andréa D.; BARROS, Aluísio JD; HALLAL, Pedro C. Generic drugs in Brazil: known by many, used by few. Cadernos de Saúde Pública, v. 21, n. 6, p. 18081815, 2005. Disponível:<http://www.scielo.br/pdf/csp/v21n6/19.pdf> Acesso em: 10 out. 2018.

BLATT, Carine R. et al. Conhecimento popular e utilização dos medicamentos genéricos na população do município de Tubarão, SC. Revista Ciência e Saúde Coletiva, Rio de Janeiro, v.17,n.p.79-87,Jan.2012.Disponível em:<http:/ /www.scielo. br/scielo.php?script=sci_arttext\&pid=S1413-81232012000100011\&lng=en\&nrm=iso $>$. Acesso em 18 out. 2018.

BRASIL. Ministério da Saúde. Portaria n. 3.916 de 30 de outubro de 1998. Dispõe sobre a Política Nacional de Medicamentos. Diário Oficial da República Federativa do Brasil. Brasília, DF, 2001. Disponível em:<http:/ /bvsms.saude.gov.br/bvs/ saudelegis/gm/1998/prt3916_30_10_1998.html> Acesso em: 6 out. 2018.

BRASIL. Agência Nacional de Vigilância Sanitária. Lei n ${ }^{\circ}$ 9787, de 10 de fevereiro de 1999. Altera a Lei $n^{\circ}$ 6360, de 23 de setembro de 1976, que dispões sobre a vigilância 
sanitária, estabelece o medicamento genérico, dispõe sobre a utilização de nomes genéricos em produtos farmacêuticos e dá outras providências. Diário Oficial da República Federativa do Brasil, Brasília, DF, 1999. Disponível em:<http:/ / www. planalto.gov.br/ccivil_03/leis/L9787.htm> Acesso em: 5 out. 2018.

BRASIL. Ministério da Saúde. Agência Nacional de Vigilância Sanitária. ResoluçãoRDC no. 58, de 10 de outubro de 2014. Dispõe sobre as medidas a serem adotadas junto à Anvisa pelos titulares de registro de medicamentos para a intercambialidade de medicamentos similares com medicamento de referência. Diário Oficial da

República Federativa do Brasil, Brasília, DF, 13 out. 2014. Seção 1, p. 660. Disponível em:<http://bvsms.saude.gov.br/bvs/saudelegis/anvisa/2014/rdc0058_10_10_2014. pdf $>$ Acesso em: 30 out. 2018.

DA SILVA, Antonio Carlos et al. Conhecimento de um grupo de pacientes sobre medicamentos genéricos por eles utilizados. Cogitare Enfermagem, v. 12, n. 4, 2007. Disponível em:<https:/ / revistas.ufpr.br/cogitare/article/view/10067/6920> Acesso em: 15 out. 2018.

DUQUE, Marco; ROCHA, Clara; BALTEIRO, Jorge. Adesão dos utentes aos medicamentos genéricos. Revista Portuguesa de Saúde Pública, v. 32, n. 2, p. 181187, 2014. Disponível em:<https://www.sciencedirect.com/science/article/pii/ S0870902514000480> Acesso em: 12 out. 2018.

FARIA, Marta A. da S.; TAVARES-NETO, José. Conhecimento popular sobre medicamento genérico em um Distrito Docente-Assistencial do Município de Rio Branco, Estado do Acre, Brasil. Epidemiologia e Serviços de Saúde, Brasília, v. 15, n. 3, p. 37-45, set. 2006. Disponível em: <http://scielo.iec.gov.br/scielo.php?script=sci_ arttext\&pid=S1679-49742006000300005\&lng=pt\&nrm=iso $>$. Acesso em 17 out. 2018.

GERHARDT, Tatiana E; SILVEIRA, Denise T. Métodos de Pesquisa. $1^{\text {a }}$ Ed. Porto Alegre: Editora da UFRGS, 2009. Disponível em: <http:/ / www.ufrgs.br/cursopgdr/ downloadsSerie/derad005.pdf> Acesso em: 30 out. 2018.

GUTTIER, Marília C. et al. Percepção, conhecimento e uso de medicamentos genéricos no Sul do Brasil: o que mudou entre 2002 e 2012?. Cadernos de Saúde Pública, v. 32, p. e00070215, 2016. Disponível em:<http://www.scielo.br/pdf/csp/v32n7/16784464-csp-32-07-e00070215.pdf> Acesso em: 15 out. 2018.

GUTTIER, Marília C. et al. Factors influencing the preference for purchasing generic drugs in a Southern Brazilian city. Revista de Saúde Pública, São Paulo, v. 51, 59, 2017. Disponível em: <http://www.scielo.br/scielo.php?script=sci arttext\&pid=S0034-89102017000100253\&lng=en\&nrm=iso $>$ Acesso em: 18 out. 2018.

LIRA, Claudio A. B. de et al. Conhecimento, percepções e utilização de medicamentos genéricos: um estudo transversal. Einstein, São Paulo, v. 12, n. 3, p. 267-273, Sept. 2014. Disponível em:<http:/ / www.scielo.br/scielo.php?script=sci_ arttext\&pid=S1679-45082014000300267\&lng=en\&nrm=iso $>$. Acesso em: 18 out. 2018. 
MARCONI, Marina de Andrade; LAKATOS, Eva Maria. Fundamentos de metodologia científica. 5. ed.-São Paulo: Atlas, 2003.

MONTEIRO, Camila N. et al. Utilização de medicamentos genéricos no município de São Paulo em 2003: estudo de base populacional. Epidemiologia e Serviços de Saúde, Brasília, v. 25, n. 2, p. 251-258, June 2016. Disponível em:<http:/ / www.scielo. $\mathrm{br} /$ scielo.php?script=sci_arttext\&pid=S2237-96222016000200251\&lng=en\&nrm=iso $>$ Acesso em: 18 out. 2018.

ROCHA, Chiara Erminia da; BARROS, José Augusto Cabral de; SILVA, Maria Dolores Paes. Levantamento de dados sobre o conhecimento e informação acerca dos medicamentos genéricos em uma população de pacientes do serviço de saúde ambulatorial do Recife, Pernambuco, Brasil. Cadernos de Saúde Pública, Rio de Janeiro, v. 23, n. 5, p. 1141-1150, May 2007. Disponível em:<http:/ / www.scielo.br/ scielo.php?script=sci_arttext\&pid=S0102-311X2007000500016> Acesso em: 10 out. 2018.

SEWELL, Keri et al. Peer Reviewed: Perceptions of and Barriers to Use of Generic Medications in a Rural African American Population, Alabama, 2011. Preventing chronic disease, v. 9, 2012. Disponível em:<https:/ / www.ncbi.nlm.nih.gov/pmc/ articles/PMC3475503/?tool=pubmed> Acesso em: 12 out. 2018.

VOSGERAU, Milene Zanoni da Silva; SOUZA, Regina Kazue Tanno de; SOARES, Darli Antonio. Utilização de genéricos em área de atuação da equipe de Saúde da Família em município do sul do Brasil. Revista Brasileira de Epidemiologia, São Paulo, v. 14, n. 2, p. 253-263, June 2011. Disponível em:<http:/ / www.scielo.br/scielo. php?script $=$ sci_arttext\&pid=S1415-790X2011000200007\&lng $=$ en\&nrm $=$ iso $>$. Acesso em: 16 out. 2018. 